\title{
Minimization of the influence of passive-light contribution in active imaging of the degree of polarization
}

\author{
Arnaud Bénière, ${ }^{1,2, *}$ François Goudail, ${ }^{1}$ Mehdi Alouini, ${ }^{2}$ and Daniel Dolfi ${ }^{2}$ \\ ${ }^{1}$ Laboratoire Charles Fabry de l'Institut d'Optique, CNRS, Université Paris-Sud, Campus Polytechnique, RD 128, \\ 91127 Palaiseau, France \\ ${ }^{2}$ Thales Research and Technology-France, RD128, 91767 Palaiseau Cedex, France \\ *Corresponding author: arnaud.beniere@institutoptique.fr
}

Received July 7, 2008; revised August 27, 2008; accepted September 6, 2008; posted September 15, 2008 (Doc. ID 97943); published October 13, 2008

Active imaging systems that measure the degree of polarization (DOP) are often perturbed by passive light owing to ambient illumination. Passive light introduces a shot noise that combines with the noise due to the active signal to perturb estimation of the DOP. We quantitatively study its influence and show that the polarization state of active illumination can be adjusted to minimize the influence of passive light. It is thus an additional degree of freedom for optimization. () 2008 Optical Society of America

OCIS codes: $260.543,030.4280$.

A simple mode of active degree of polarization (DOP) imaging consists in illuminating the scene with purely polarized light and analyzing the light backscattered in the direction of the detector with analyzers respectively parallel and orthogonal to the incident state of polarization. This finds applications in biomedicine [1], remote sensing [2], or imaging through turbid media [3]. However, as is the case with every active imagery technique, it is often perturbed by passive contribution owing to ambient light. This contribution is usually estimated by turning off the active illumination and acquiring an image that is then subtracted from the active one. However, the noise in the subtracted passive image perturbs the estimation of the DOP. Moreover, the passive contribution is often considered totally depolarized in active systems, which is not actually the case. For instance, ambient light refracted by objects [4] or scattered in turbid medium [5] is partially polarized. Our purpose in this Letter is to model precisely this phenomenon and propose ways to optimize the polarization state of active illumination to minimize this effect.

We will assume that the only source of noise in the passive and active images is photon shot noise, which means that the imaging system reaches its fundamental limit. The scene is illuminated with a light polarized in any state on the Poincaré sphere. A first image $X$, is formed with the fraction of light scattered in the same polarization state as the incident light. A second image $Y$ is formed with the light scattered in a polarization state orthogonal to the incident one. We consider subsamples $X_{i}$ and $Y_{i}, i \in[1, N]$ of the images, that may be spatial if we consider a small set of neighboring pixels or temporal if we consider a single pixel in several successive acquisitions. To permit rigorous treatment of estimation properties, each sample is assumed homogeneous, that is, the average number of photoelectrons is the same for all pixels. If this property is not fulfilled, the effect will be application dependent. For example, in target detection, inhomogeneity of the sample would decrease the probability of detection. Measurements $X_{i}$ and $Y_{i}$ are expressed in number of photoelectrons and can be gathered in an $N$-sized statistical sample $\chi=\{\mathbf{X}, \mathbf{Y}\}$. They are both random variables that follow Poisson distributions of mean values $\left(m_{X}+g_{X}\right)$ and $\left(m_{Y}+g_{Y}\right)$, respectively. Here, $m_{X}$ and $m_{Y}$ represent average values of the useful signal coming from active illumination scattered by the scene. Parameters $g_{X}$ and $g_{Y}$ correspond to the average number of photoelectrons due to passive contribution (Fig. 1). To represent the useful signal, we will rather use the following parametrization:

$$
I=m_{X}+m_{Y}, \quad P=\frac{m_{X}-m_{Y}}{m_{X}+m_{Y}}
$$

where $I$ is the total intensity of the useful signal and $P$ is the parameter of interest. It is often improperly called the degree of polarization. If the observed materials are purely depolarizing, that is, if the principal polarization state of the scattered light is that of the illuminating light, then $P$ is the actual DOP of the scattered light, which has the property $P \in[0,1]$ [4]. This assumption is reasonable for natural materials and turbid media observed in monostatic (backscattering) configuration with linearly polarized illumination [2]. However, if, for instance, the illumination state is circular, backscattered light has reversed helicity, and $P$ is negative. To avoid ambigu-

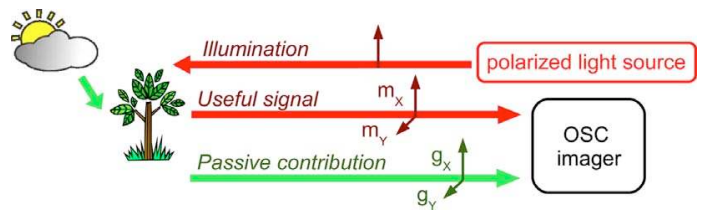

Fig. 1. (Color online) Imaging configuration. The useful signal corresponds to active illumination scattered by the scene. The passive contribution is due to scattered ambient light. 
ity, we will call $P$ the orthogonal state contrast (OSC). In all cases, $P \in[-1,1]$ and provides a useful information about the scene.

Our objective is to estimate $I$ and $P$ (or $m_{X}$ and $m_{Y}$ ) from the data. It is easily seen that the observation of $\chi$ is not sufficient for that purpose. A separate data set is required to estimate $g_{X}$ and $g_{Y}$. Usually, the illumination is switched off, and passive images $V_{i}$ $\left(W_{i}\right), i \in[1, N]$ are formed with the analyzer parallel (orthogonal) to the incident polarization state. The average photoelectron number in this image is $g_{X}$ $\left(g_{Y}\right)$ and is perturbed by Poisson noise. We thus finally obtain a statistical sample $\chi^{\prime}=\{\mathbf{X}, \mathbf{Y}, \mathbf{V}, \mathbf{W}\}$ composed of four random vectors whose elements follow independent Poisson distributions of respective mean values $m_{X}+g_{X}, m_{Y}+g_{Y}, g_{X}$, and $g_{Y}$.

Our objective is to determine the precision of estimation of the OSC parameter $P$ from this sample. For that purpose, we determine the Cramer-Rao lower bound (CRLB) [6], which is a lower bound on the variance that can be reached by unbiased estimators. It is thus a standard way to quantify the intrinsic difficulty of an estimation problem, since it gives a good order of magnitude of the expected precision. According to the above-defined statistical data model, the expression of the likelihood is

$$
\begin{aligned}
\ell\left(I, P, g_{X}, g_{Y}\right)= & -N\left(I+2\left(g_{X}+g_{Y}\right)\right)+A \\
& +S_{X} \log \left[\frac{I(1+P)}{2}+g_{X}\right]+S_{V} \log \left(g_{X}\right) \\
& +S_{Y} \log \left[\frac{I(1-P)}{2}+g_{Y}\right]+S_{W} \log \left(g_{Y}\right),
\end{aligned}
$$

where $S_{U}=\sum_{i=1}^{N} U_{i}$, with $U=\{X, Y, V, W\}$, and $A$ is independent of $I, P, g_{X}$, and $g_{Y}$. The vector of parameters to estimate is thus $\theta^{T}=\left[I, P, g_{X}, g_{Y}\right]$. From Eq. (1), one can calculate the Fisher matrix, defined as $F_{i j}=-\left\langle\partial \ell / \partial \theta_{i} \partial \theta_{j}\right\rangle$, where $\langle$.$\rangle denotes statistical averag-$ ing, and invert it. By definition, the CRLB on the four parameters are the diagonal elements of the matrix $F^{-1}$. After cumbersome but elementary computations, one obtains the CRLB for the three nuisance parameters: $\kappa_{g_{X}}=g_{X} / N, \quad \kappa_{g_{Y}}=g_{Y} / N, \quad$ and $\kappa_{I}=\left(I+2 I_{g}\right) / N$, where $I_{g}=g_{X}+g_{Y}$ is the total number of passive photoelectrons. The CRLB on $g_{X}$ and $g_{Y}$ corresponds to the estimation variance of the mean of a Poisson random variable. The CRLB on $I$ is the sum of two contributions: the first term corresponds to the contribution of the useful signal, and the second to that of the passive light. Finally, the CRLB on the parameter $P$ is found to be $\kappa_{P}=\kappa_{P}^{u}+\kappa_{P}^{p}$, with

$$
\kappa_{P}^{u}=\frac{\left(1-P^{2}\right)}{N I} \quad, \kappa_{P}^{p}=2 I_{g} \frac{1+P^{2}-2 P_{g} P}{N I^{2}},
$$

where $P_{g}=\left(g_{X}-g_{Y}\right) /\left(g_{X}+g_{Y}\right)$ is the OSC of the passive light. This CRLB is also the sum of two terms: $\kappa_{P}^{u}$ corresponds to the shot noise of the useful signal, $\kappa_{P}^{p}$ to that of the passive contribution. It is interesting to notice that $\kappa_{P}^{p}$ depends not only on the total number of passive photoelectrons $I_{g}$ but also on their OSC $P_{g}$. This means that for a given total number $I_{g}$ of passive photoelectrons, their repartition between the two polarization channels influences the potential precision of estimation of the OSC. Let us consider different cases. If $P_{g}=0$, that is, $g_{X}=g_{Y}=g, \kappa_{P}^{p}$ has twice the value determined in [7]. This is due to the fact that $g$ has been assumed unknown in the present article, whereas it was assumed perfectly known in [7]. The price to pay for not knowing its value and having to estimate it is to double the estimation variance. If $\left|P_{g}\right|=1$ and $P_{g}$ has the same sign as $P$ (passive light is purely polarized parallel to the state in which most useful photons are scattered), then the CRLB is minimal (and the estimation precision is best). Conversely, if $\left|P_{g}\right|=1$ and the sign of $P_{g}$ is the opposite of that of $P$, then the CRLB is maximal. It is thus better to have most passive photons in the polarization channel where most useful photons are measured. The difference between the maximal and the minimal value of the CRLB as $P_{g}$ varies between +1 or -1 is $\Delta \kappa=8\left(I_{g} P\right) /\left(N I^{2}\right)$. It is maximal when $P$ is large, that is, when the useful light is polarized. If it is depolarized $(P=0)$, then $P_{g}$ has no influence of the estimation precision of $P$.

We have represented in Fig. 2 the variation of the square root of $\kappa_{P}$ (equivalent to a standard deviation) as a function of the intensity of the useful signal $I$ for $P=0.9, I_{g}=500$, and $P_{g}=1,0$ and -1 . This situation corresponds to a low-flux imaging application. The curves are clearly constituted of two parts, in which the dominant source of noise is different. When the object is far, $I$ is small, the noise due to passive contribution is dominant, and the curves depend on the value of $P_{g}$. When $I$ becomes larger, shot noise fluctuations due to the useful signal are dominant and the curves tend to join together: estimation performance no longer depends on $P_{g}$.

The previous analysis is based on the CRLB, which is a potential estimation precision. It is now interesting to study actual estimators of the OSC. Since we know the expression of the likelihood [Eq. (1)], we can use the profile likelihood method, which considers all parameters as parameters of interest and estimates them in the maximum likelihood sense. For that purpose, we set to zero the derivatives of the loglikelihood with respect to each parameter and solve

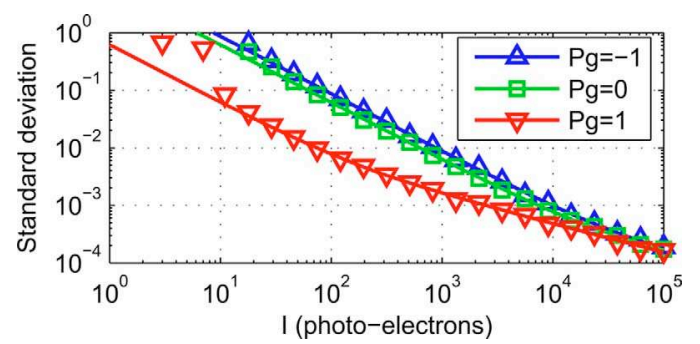

Fig. 2. (Color online) Square root of the CRLB $\kappa_{P}$ (solid curves) and estimated standard deviation of the profile likelihood estimator $\hat{P}_{p l}$ (markers) on $10^{5}$ realizations for three different values of $P_{g}$ as a function of the intensity $I$, with $I_{g}=500$ photoelectrons, $P=0.5$, and $N=50$. 
the obtained set of equations. One obtains the following estimate of $P$ :

$$
\hat{P}_{p l}=\frac{\hat{m}_{X}-\hat{m}_{Y}}{\hat{m}_{X}+\hat{m}_{Y}},
$$

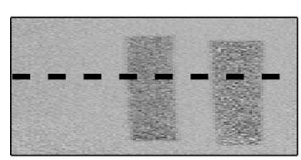

(a)

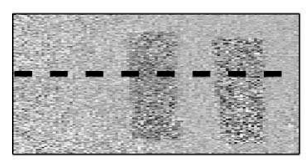

(c)

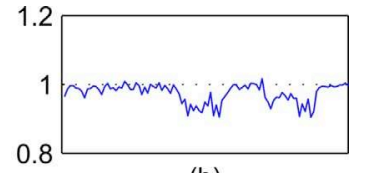

(b)

(d)

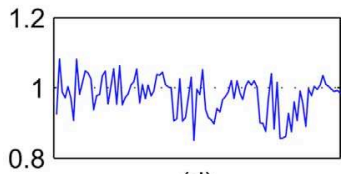

rather intuitive, since it consists in estimating $m_{X}$ $\left(m_{Y}\right)$ by subtracting the estimate of $g_{X}\left(g_{Y}\right)$ from the sample values then calculating their empirical mean and forming the OSC. The interesting result is that, for the noise model at hand, this estimator is actually the profile likelihood estimator and thus benefits from the good properties of this type of estimator. We have represented in Fig. 2 the estimated standard deviation of $\hat{P}_{p l}$ for different values of $P_{g}$. It is seen that in the considered case, for $I>10$ photoelectrons, this estimator reaches the CRLB and can be considered efficient. Its variance becomes larger than the CRLB for smaller values of $I$. This is due to the form of the estimator: estimates of $g_{U}$ are subtracted from the data, and when noise on $\hat{g}_{U}$ is high, the value of the denominator can be close to zero, leading to very large variations. To validate these results, we have performed the following experiment that reproduces the situation of Fig. 1. A first polarizer is fixed in front of a light source. The resulting beam is linearly polarized and constitutes the "active" illumination, leading to the useful signal. A second polarizer is placed in front of another light source that produces the "passive" illumination. The level of this second source is such that we are in the passive noisedominant regime. The second polarizer is mounted on a rotating plate so that it can produce light linearly polarized in different directions. Images are acquired with an OSC imager based on a Basler A312f 12 bit camera. We consider two configurations: in the first one, $P_{g} \simeq 1$, which means that the state of polarization of the passive light is parallel to that of active light. In the other configuration, $P \simeq-1$, namely, the polarization states of the passive and active illuminations are orthogonal. The observed scene consists of two transparent plastic ribbons appearing on an aluminium plate. The OSC of the light scattered by the two types of materials are high: $P \simeq 1$ for the plate and $P \simeq 0.9$ for the plastic ribbons. We have represented in Fig. 3 the images observed in the two configurations. OSC image is significantly noisier when $P_{g} \simeq-1$ [Fig. 3(c)] than when $P_{g} \simeq 1$ [Fig. 3(a)]. This observation is verified in the profiles plotted along a line in Figs. 3(b) and 3(d); the two plastic ribbons are hardly detectable when $P_{g} \simeq-1$. The values of the estimation variances of $P$ on the two types of materials are in good agreement with the corresponding CRLB. As an example the estimated variance on the aluminium with $P_{g} \approx 1$ is $3.6 \times 10^{-4}$ and the corresponding CRLB is $3.5 \times 10^{-4}$.

Fig. 3. (Color online) OSC image of a scene composed of two transparent plastic ribbons on an aluminium plate with (a) $P_{g} \approx 1$ configuration and (c) $P_{g} \approx-1$ configuration. The respective profile of the OSCI along the dotted line is plotted in (b) and (d).

These results show that taking into account the polarization state of the passive contribution offers a way to improve the estimation (and detection) efficiency. If the scene is purely depolarizing (which is a good approximation for natural scenes), and $P$ is positive, the optimal choice consists in minimizing $\kappa_{p}$ [Eq. (2)] by illuminating with the principal polarization state of the passive contribution. For non-purelydepolarizing scenes, $P$ may vary with the illumination as well as $\kappa_{P}$. In this case, one may seek to maximize the contrast, which is an applicationdependent function of both $P$ and $\kappa_{P}$.

We have analyzed the influence of passive light on precision of estimation of OSC in active images when the only source of noise is photon shot noise. This study shows that the state of polarization of the spurious ambient light must be taken into account in order to maximize the precision of estimation. The polarization state of illumination becomes then an additional degree of freedom in optimizing the sensitivity of active polarimetric imaging systems. It controls the limit of detection in low signal-to-noise-ratio imaging scenarios in the visible and IR bands.

Arnaud Bénière's Ph.D thesis is supported by the Délégation Générale pour l'Armement, MRIS domain IMAT (contact: Jacques Blanc-Talon).

\section{References}

1. M. Anastasiadou, A. De Martino, D. Clement, F. Lige, B. Laude-Boulesteix, N. Quang, J. Dreyfuss, B. Huynh, A. Nazac, L. Schwartz, and H. Cohen, Phys. Status Solidi C 5, 991 (2008).

2. S. Breugnot and P. Clémenceau, Opt. Eng. 39, 2681 (2000).

3. R. E. Nothdurft and G. Yao, Appl. Opt. 45, 5532 (2006).

4. C. Brosseau, Fundamentals of Polarized Light-a Statistical Approach (Wiley, 1998).

5. P. C. Y. Chang, J. C. Flitton, K. I. Hopcraft, E. Jakeman, D. L. Jordan, and J. G. Walker, Appl. Opt. 42, 2794 (2003).

6. S. M. Kay, Fundamentals of Statistical Signal Processing-Volume I: Estimation Theory (PrenticeHall, 1993).

7. A. Bénière, F. Goudail, M. Alouini, and D. Dolfi, J. Eur. Opt. Soc. Rapid Publ. 3, 08002 (2008). 